Revisión

\title{
Diagnóstico de muerte encefálica
}

\author{
DOLORES ESCUDERO \\ Servicio de Medicina Intensiva. Hospital Universitario Central de Asturias. Oviedo. Asturias. España.
}

La muerte encefálica ha sido reconocida como la muerte del individuo por la comunidad científica y aceptada como tal en la legislación de diferentes países. La muerte encefálica se define como el cese irreversible en las funciones de todas las estructuras neurológicas intracraneales, tanto de los hemisferios cerebrales como del troncoencéfalo. Esta situación aparece cuando la presión intracraneal supera la presión arterial sistólica del paciente, lo que da lugar a la parada circulatoria cerebral. La etiología más frecuente es la hemorragia cerebral y el traumatismo craneoencefálico. El diagnóstico debe ser realizado por médicos expertos en el manejo de pacientes neurocríticos y se basa en una exploración neurológica completa y extremadamente rigurosa que constate un coma arreactivo y ausencia de reflejos troncoencefálicos y respiración espontánea. Las pruebas instrumentales pueden ser obligatorios en algunos casos y varían en cada país. Los de tipo electrofisiológico son el electroencefalograma y los potenciales evocados. En pacientes tratados con fármacos sedantes se utilizan pruebas que evalúan el flujo sanguíneo cerebral, como la angiografía cerebral, el Doppler transcraneal y la gammagrafía con ${ }^{99}$ Tc-HMPAO.

En España, más del $92 \%$ de los trasplantes se realizan con órganos procedentes de donantes en muerte encefálica. La declaración de muerte encefálica es un acto de gran responsabilidad, con trascendencia médica, ética y legal, ya que exige retirar todas las medidas artificiales de soporte o realizar la extracción de órganos para trasplante.

Correspondencia: Dra. D. Escudero.

Servicio de Medicina Intensiva. Hospital Universitario Central de Asturias.

Celestino Villamil, s/n. 33006 Oviedo. Asturias. España.

Correo electrónico: dolores.escudero@sespa.princast.es

lolaescudero@telefonica.net

Manuscrito aceptado el 26-5-2008
Un amplio conocimiento sobre su diagnóstico y una correcta toma de decisiones evitan el consumo innecesario de recursos y optimizan la obtención de órganos para trasplante.

PALABRAS CLAVE: Muerte encefálica. Donante de órganos. Doppler transcraneal. Angiografía por tomografía computarizada. Gammagrafía cerebral con ${ }^{99} \mathrm{Tc}-\mathrm{HMAO}$.

\section{BRAIN DEATH DIAGNOSIS}

Brain death has been recognized by the scientific community as the person's death, and accepted in the legislation of different countries. Brain death is defined as the irreversible ending of the functions of all the intracranial neurological structure in both the brain and brain stem. This clinical situation appears when intracranial pressure exceeds the patient's systolic blood pressure, leading to brain circulatory arrest. The most frequent are cerebral hemorrhage and cranioencephalic trauma. Clinical diagnostic must be done by doctors with expertise in neurocritical patient treatment. This diagnosis is based on a systematic, complete and extremely rigorous clinical examination that confirms a non-reactive coma, absence of brain stem reflex, and absence of spontaneous breathing. Instrumental tests may be obligatory in some cases, this depending on each country. Electroencephalogram and evoked potentials are the electrophysiological tests used. In patients treated with sedative drugs, cerebral blood flow evaluation tests, such as cerebral angiography, transcranial Doppler or ${ }^{99} \mathrm{Tc}-\mathrm{HMPAO}$ scintigraphy, will be used.

More than $92 \%$ of the transplants performed in Spain are performed with brain death donor organs. Brain death confirmation is a high responsibility act, with medical, ethical and legal significance since it requires removal of all artificial support, or organs extraction for transplant. Extensive knowledge on its diagnostic and cor- 
rect decision making avoid unnecessary use of resources and improves management of organs for transplant.

KEY WORDS: Brain death. Organ donor. Transcranial Doppler. Computed tomography angiography. Scintigraphy with ${ }^{99} \mathrm{Tc}$ HMPAO.

\section{INTRODUCCIÓN}

El concepto de muerte siguiendo criterios neurológicos, lo que se conoce como muerte encefálica (ME), ha sido reconocido como la muerte del individuo por la comunidad científica y aceptado como tal en la legislación de diferentes países ${ }^{1,2}$.

La ME se define como el cese irreversible en las funciones de todas las estructuras neurológicas intracraneales, tanto de los hemisferios cerebrales como del troncoencéfalo. Esta situación clínica aparece cuando la presión intracraneal (PIC) se eleva por encima de la presión arterial sistólica (PAS) del paciente, lo que da lugar a la parada circulatoria cerebral.

La etiología de la ME incluye los siguientes cuadros: ictus isquémico o hemorrágico, hemorragia subaracnoidea, traumatismo craneoencefálico (TCE), encefalopatía anóxica, infecciones y tumores del sistema nervioso central (SNC). En nuestro medio, la causa más frecuente es la hemorragia cerebral intraparenquimatosa de origen hipertensivo. La afección neurológica que causa ME tiene, en la inmensa mayoría de los casos, una localización supratentorial.

En España, alrededor del 14\% de los pacientes que fallecen en las unidades de cuidados intensivos lo hacen en situación de ME, y pueden llegar a ser el 30\% si la UCI es centro de referencia de neurocirugía ${ }^{3,4}$ La declaración de ME es un diagnóstico de gran responsabilidad, con trascendencia médica, ética y legal, ya que exige retirar todas las medidas artificiales de soporte -incluida la ventilación mecánica- o realizar la extracción de órganos para trasplante.

El diagnóstico de ME se basa en una exploración neurológica exhaustiva que debe ser realizada por médicos expertos en el manejo de pacientes neurocríticos. Además del diagnóstico clínico, hay una serie de pruebas instrumentales que pueden ser obligatorias en algunos casos y varían de un país a otro. La legislación española define perfectamente las circunstancias clínicas que obligan a utilizar el diagnóstico instrumental ${ }^{1}$.

En la actualidad, más del $92 \%$ de los trasplantes que se realizan en España se hacen con órganos procedentes de donantes en ME. Estos datos, similares a los de otros países, demuestran la importancia de la ME como entidad generadora de órganos para trasplante y la convierten en una de las piezas indispensables del proceso donación-trasplante.

Un amplio conocimiento sobre el diagnóstico de ME y una correcta toma de decisiones evita el consumo innecesario de recursos y optimiza la obtención de órganos para trasplante.

\section{DIAGNÓSTICO CLÍNICO}

La exploración neurológica del paciente en el que se sospecha ME tiene que ser realizada por médicos expertos en la valoración de pacientes con estado neurológico crítico. Esta exploración debe ser sistemática, completa y extremadamente rigurosa ${ }^{5-11}$. Dada la importancia clínica y legal del diagnóstico, todos los hallazgos obtenidos, así como la hora de la exploración, deben reflejarse adecuadamente en la historia clínica. Para realizar el diagnóstico clínico de ME han de cumplirse las siguientes condiciones.

\section{Coma estructural de etiología conocida y carácter irreversible}

Es imprescindible conocer la causa del coma mediante la historia clínica y/o la documentación por neuroimagen con tomografía computarizada (TC) craneal que demuestre lesión estructural grave y catastrófica en el sistema nervioso central.

\section{Requisitos clínicos}

Antes de iniciar la exploración neurológica, hay que comprobar que el paciente se encuentra en condiciones clínicas adecuadas que no alteren los hallazgos de la exploración clínica. Estos requisitos, considerados básicos en todos los protocolos internacionales, son: a) estabilidad cardiocirculatoria; b) oxigenación y ventilación adecuadas, y c) ausencia de hipotermia grave. La homeostasis térmica depende de la activación del hipotálamo anterior, que es el integrador común de la información aferente y eferente. En ME, al perderse la función de este centro termorregulador, se produce una hipotermia espontánea y progresiva. En los criterios de la Academia Americana de Neurología y en la legislación española, se acepta como válida una temperatura superior a $32{ }^{\circ} \mathrm{C}$. Entre 28 y $20{ }^{\circ} \mathrm{C}$, la propia hipotermia hace que el paciente pueda estar comatoso y con abolición de reflejos troncoencefálicos, en una situación neurológica que puede asemejarse a la ME. Aunque se acepta como válida una temperatura superior a $32{ }^{\circ} \mathrm{C}$, es preferible mantener una temperatura siempre superior a $35^{\circ} \mathrm{C}$ para evitar los efectos clínicos indeseables de la propia hipotermia y facilitar la realización del test de apnea, ya que si el paciente está hipotérmico, se genera poco $\mathrm{CO}_{2}$, lo que dificulta alcanzar cifras de $\mathrm{PaCO}_{2}>60$ $\mathrm{mmHg}$.

\section{Ausencia de alteraciones metabólicas}

Hay que descartar alteraciones metabólicas o tóxicas importantes hidroelectrolíticas, acidobásicas o endocrinas, tales como hipofosfatemia grave, encefalopatía hepática, coma hipoglucémico o coma hipotiroideo. 


\section{Ausencia de intoxicaciones}

Los tóxicos depresores del SNC pueden motivar graves errores en la valoración neurológica, por lo que es obligatorio descartar la presencia de dichas sustancias mediante una exhaustiva historia clínica y determinaciones en sangre y orina.

\section{Ausencia de fármacos depresores del sistema nervioso central}

En el tratamiento de los pacientes neurocríticos se utilizan habitualmente benzodiacepinas, propofol, opiáceos y barbitúricos que pueden enmascarar la exploración neurológica. Los barbitúricos a dosis elevadas y mantenidas llegan a producir coma profundo sin respuesta a estímulos algésicos, ausencia de reflejos troncoencefálicos y EEG sin actividad bioeléctrica cerebral ${ }^{12-14}$. El pentobarbital y el tiopental son barbitúricos de acción corta y ultracorta de efectos similares que se diferencian sólo en su farmacocinética. El pentobarbital se metaboliza en el hígado; la velocidad de inactivación hepática por hidroxilación es de un $0,5 \% / \mathrm{h}$ y su vida media es de unas $22-60 \mathrm{~h}$. El tiopental se une a las proteínas en un $65-85 \%$ y se metaboliza en el hígado a razón de un $10-15 \% / h$ mediante oxidación a ácido carboxílico (metabolito inactivo) o mediante disulfuración a pentobarbital (metabolito activo). A dosis anestésicas, el tiopental sigue una cinética de eliminación lineal y su vida media es de 6-8 h, pero a dosis elevadas y en tratamientos prolongados, como las utilizadas en el coma barbitúrico, su vida media se prolonga notablemente, y de forma muy variable, entre 6 y 60 h, con una cinética de tipo no lineal o de orden cero por saturación de las enzimas encargadas de su degradación. La edad, los factores individuales y las alteraciones hemodinámicas y hepáticas pueden prolongar su vida media.

En los pacientes tratados con barbitúricos no se podrá valorar la exploración neurológica. Dadas las características farmacológicas del tiopental y la escasez de estudios con series amplias sobre su uso prolongado y a dosis elevadas, es muy difícil poder definir de forma exacta cuántas horas se ha de esperar tras la suspensión del tratamiento para aceptar como válidos los hallazgos neurológicos, ya que, además de la variabilidad individual, concurren muchos y variados factores, algunos de ellos no suficientemente conocidos en la actualidad ${ }^{15,16}$. Cordato et al $^{17}$ estudiaron a 75 pacientes en tratamiento con barbitúricos en las dosis necesarias para obtener un patrón salvasupresión en el EEG y refieren que el reflejo fotomotor se recupera con concentraciones plasmáticas de thiopental de $50 \mathrm{mg} / \mathrm{l}$ y las respuestas motrices, con valores de $12 \mathrm{mg} / \mathrm{l}$, pero encuentran grandes variaciones interindividuales en el tiempo de recuperación de ambas respuestas.

Los bloqueadores neuromusculares producen una paralización muscular que negativiza las respuestas motrices al estímulo algésico y algunos reflejos troncoencefálicos, por lo que hay que asegurarse de que no han sido administrados antes de realizar la exploración neurológica o realizar una comprobación mediante un estimulador de nervio periférico.

En caso de sospecharse enclavamiento cerebral por modificaciones pupilares, mayor inestabilidad hemodinámica, poliuria por diabetes insípida neurogénica o cambios en los datos de neuromonitorización (antecedentes de PIC superiores a la presión de perfusión cerebral de forma mantenida, ascenso de $\mathrm{SJO}_{2}$ a valores por encima del $90 \%$ ), para realizar el diagnóstico de ME será preciso utilizar métodos instrumentales de flujo como la angiografía cerebral en sus diversas modalidades, la gammagrafía cerebral con ${ }^{99} \mathrm{Tc}-\mathrm{HMPAO}$ o el Doppler transcraneal.

\section{Exploración neurológica}

El diagnóstico clínico de ME se basa en tres pilares fundamentales: coma arreactivo, ausencia de reflejos troncoencefálicos y apnea.

\section{Coma arreactivo}

El paciente debe presentar hipotonía muscular generalizada, coma profundo y arreactivo con nivel 3 en la Escala de Coma de Glasgow. La estimulación algésica se realiza en el territorio de los nervios craneales (trigémino), provocando dolor intenso a nivel supraorbitario, del labio superior o en la articulación temporomandibular. No puede haber ningún tipo de respuesta motriz o vegetativa, como tampoco respuestas o actitudes motrices en descerebración ni descorticación.

\section{Ausencia de reflejos troncoencefálicos}

La exploración debe ser bilateral y se debe constatar la ausencia de todos los reflejos.

Reflejo fotomotor. En ME las pupilas pueden ser redondas, ovales o discóricas y de tamaño medio o midriáticas, desde 4 a $9 \mathrm{~mm}$, pero siempre son arreactivas a la luz. Tampoco debe haber respuesta consensual. Vía aferente, nervio óptico. Vía eferente, nervio motor ocular común. Nivel anatómico explorado: mesencéfalo.

Reflejo corneal. Al estimular la córnea con una gasa no se observa contracción palpebral, enrojecimiento o lagrimeo. Vía aferente, nervio trigémino. Vía eferente, nervio facial. Nivel anatómico: protuberancia.

Reflejo oculocefálico. Manteniendo abiertos los ojos del paciente, se realizan giros rápidos de la cabeza en sentido horizontal. En ME no se observa ningún tipo de movimiento ocular. Vía aferente, nervio auditivo. Vía eferente, nervio motor ocular común y motor ocular externo. Nivel anatómico: unión bulboprotuberancial. 
Reflejo oculovestibular. Con la cabeza a $30^{\circ}$, se inyectan en el conducto auditivo externo $50 \mathrm{ml} \mathrm{de}$ suero frío, manteniendo abiertos los ojos del paciente durante 1 min y observando en condiciones normales un nistagmo de componente lento hacia el oído irrigado y componente rápido que se aleja del conducto irrigado. El nistagmo es regular y rítmico y permanece menos de 2-3 min. En ME no se produce ningún tipo de movimiento ocular. Vía aferente, nervio auditivo. Vía eferente, nervio motor ocular común y nervio motor ocular externo. Nivel anatómico, unión bulboprotuberancial.

Reflejo nauseoso. Estimulando el velo del paladar blando, la úvula y la orofaringe, no se observa respuesta nauseosa. Vía aferente, nervio glosofaríngeo. Vía eferente, nervio vago. Nivel anatómico: bulbo.

Reflejo tusígeno. Se introduce una sonda a través del tubo endotraqueal, provocando estimulación de la tráquea. Con el fin de evitar la despresurización de la vía aérea, las aspiraciones endobronquiales deben realizarse siempre a través del diafragma del tapón del tubo traqueal. En ME no hay respuesta tusígena. Vía aferente, nervio glosofaríngeo y nervio vago. Vía eferente, nervio vago. Nivel anatómico: bulbo.

En lactantes y neonatos la exploración neurológica es más difícil de obtener debido a la propia inmadurez cerebral, por lo que se recomienda repetirla insistentemente y explorar también otros reflejos propios de la edad como el de succión y búsqueda ${ }^{18}$.

\section{Test de atropina}

Explora farmacológicamente la actividad del nervio vago y de sus núcleos troncoencefálicos. Se administran $0,04 \mathrm{mg} / \mathrm{kg}$ de sulfato de atropina intravenosa y se comprueba la frecuencia cardíaca antes y después de la inyección. La frecuencia cardíaca no debe superar el $10 \%$ de la frecuencia cardíaca basal. No se debe administrar la atropina por la misma vía venosa por la que se están infundiendo catecolaminas, ya que ello puede producir taquicardia e interferir con el resultado. El test de atropina debe realizarse después de la valoración del tamaño pupilar, ya que la atropina puede producir midriasis.

\section{Test de apnea}

Cronológicamente, debe ser realizado al final de toda la exploración. Previamente se realiza una hiperoxigenación y se modifican los parámetros del respirador para conseguir una normoventilación. Se debe extraer una gasometría arterial para documentar la $\mathrm{PaCO}_{2}$ y desconectar al paciente del respirador, introduciendo una cánula hasta la tráquea con oxigeno a $6 \mathrm{l} / \mathrm{min}$ para conseguir una oxigenación adecuada y evitar hipoxia que pueda causar asistolia. Se observará detenidamente el tórax y el abdomen comprobando que no haya ningún tipo de mo- vimiento respiratorio. Cada minuto de desconexión, la $\mathrm{PaCO}_{2}$ se eleva 2-3 mmHg. El objetivo es demostrar que el paciente no respira cuando el centro respiratorio está máximamente estimulado por hipercapnia y acidosis. Al finalizar el test de apnea se practica una gasometría arterial para constatar que la $\mathrm{PaCO}_{2}$ es $>60 \mathrm{mmHg}$.

En la actualidad no se recomienda realizar el test clásico descrito previamente ya que se produce una despresurización en la vía aérea y aumento del colapso alveolar. En todos los casos, pero especialmente cuando se trate de un potencial donante de pulmón, hay que hacer siempre un test de apnea con CPAP, aplicada mediante válvulas específicas en la salida espiratoria del tubo en T o mediante «ventilación en modo $\mathrm{CPAP}$ » en el respirador ${ }^{19}$. La mayoría de los nuevos ventiladores permiten esta modalidad de ventilación y además tienen la ventaja de que permiten visualizar en la pantalla las curvas de presión, flujo y volumen generados. La presión utilizada será de 10 $\mathrm{cmH}_{2} \mathrm{O}$, aunque puede ser más elevada según las necesidades del caso. Se puede considerar igualmente la «hipercapnia permisiva» previa para disminuir el tiempo de desconexión de la ventilación mecánica. En líneas generales, hay que saber adaptar el test de apnea a las condiciones respiratorias del paciente valorando adecuadamente la utilización de sus diferentes opciones.

\section{Actividad motriz espinal}

La actividad motriz de origen medular es un hecho observado con relativa frecuencia, por lo que es muy importante saber diferenciarla de las respuestas motrices de origen encefálico. Puede haber actividad motriz tanto espontánea como refleja. La variedad de reflejos que se observan es muy amplia y entre los más frecuentes hay que destacar los reflejos cutaneoabdominales, cremastérico, plantar flexor y extensor, de retirada y tonicocervicales. En el reflejo cervicoflexor del brazo, se observa una contracción de los músculos flexores del brazo, que suele separarse unos centímetros de la cama cuando se realiza una flexión rápida y forzada del cuello. También hay reflejos cervicoflexor de la cadera y cervicoabdominal, en cuyo caso se obtiene flexión de la cadera y contracción de la musculatura abdominal. También puede observarse actividad motriz espontánea, especialmente mioclonías espinales y actividades tónicas en las extremidades superiores que originan situaciones posturales más o menos estereotipadas. En ocasiones, pueden verse movimientos complejos como el signo de Lázaro con elevación y aproximación de ambos brazos que, en su presentación más espectacular, se acompañan de flexión del tronco hasta la sedestación.

Todas estas situaciones, sin duda muy inquietantes, requieren que se las conozca ampliamente para informar adecuadamente y tranquilizar al personal sanitario no experto, y se recomienda la administración de un bloqueador neuromuscular. 


\section{Periodo de observación}

El periodo de observación es variable en cada país. Cuando el diagnóstico de ME se realiza sólo mediante exploración neurológica, la Academia Americana de Neurología ${ }^{6}$ y la normativa española ${ }^{1}$ recomiendan periodos de observación de $6 \mathrm{~h}$ si hay daño estructural y de 24 h cuando la causa del coma es una encefalopatía anóxica. Estos periodos de observación son modificables a juicio clínico dependiendo de la etiología y la gravedad del agente causal. Si se realizan pruebas diagnósticas instrumentales, el periodo de observación puede acortarse a juicio médico.

\section{Muerte troncoencefálica «aislada»}

Es una entidad poco frecuente que aparece cuando la causa del coma es una afección de localización puramente infratentorial. En estos casos, está preservada la circulación cerebral anterior a través de las arterias carótidas internas; esta situación puede persistir durante varios días si el paciente es portador de un drenaje de líquido cefalorraquídeo. En estas circunstancias, para diagnosticar ME, además de la exploración clínica, es necesario realizar un EEG u otra prueba diagnóstica que demuestre la ausencia de función de los hemisferios cerebrales ${ }^{1,20}$.

\section{Pruebas diagnósticas instrumentales}

Las pruebas instrumentales para el diagnóstico de ME se clasifican en dos tipos: electrofisiológicas y las que evalúan el flujo sanguíneo cerebral. Un resumen del diagnóstico clínico y los métodos diagnósticos instrumentales puede verse en la tabla 1 .

\section{Electroencefalograma}

El EEG es un método diagnóstico sencillo, rápido, no invasivo y fácil de realizar a pie de cama, por lo que es el más utilizado en el diagnóstico instrumental de ME.

El EEG sólo estudia la actividad bioeléctrica de la corteza cerebral en la convexidad de los hemisferios cerebrales, pero no de la corteza de la base, la profundidad de los hemisferios ni, por supuesto, el troncoencéfalo. La principal limitación del EEG es que se interfiere por los fármacos depresores del SNC, por lo que en pacientes que reciben este tipo de tratamiento o están en coma barbitúrico no es útil. El EEG debe realizarse con una técnica rigurosa siguiendo las recomendaciones internacionales ${ }^{21}$.

\section{BIS (Bispectral Index Scale)}

La BIS es un parámetro que surge del análisis matemático complejo, multivariable (latencia, amplitud, bicoherencia) y del biespectro, extraído del EEG. La BIS se utiliza en las UCI para determinar el grado de sedación. Tiene una escala de 0 a 100 . Una BIS de

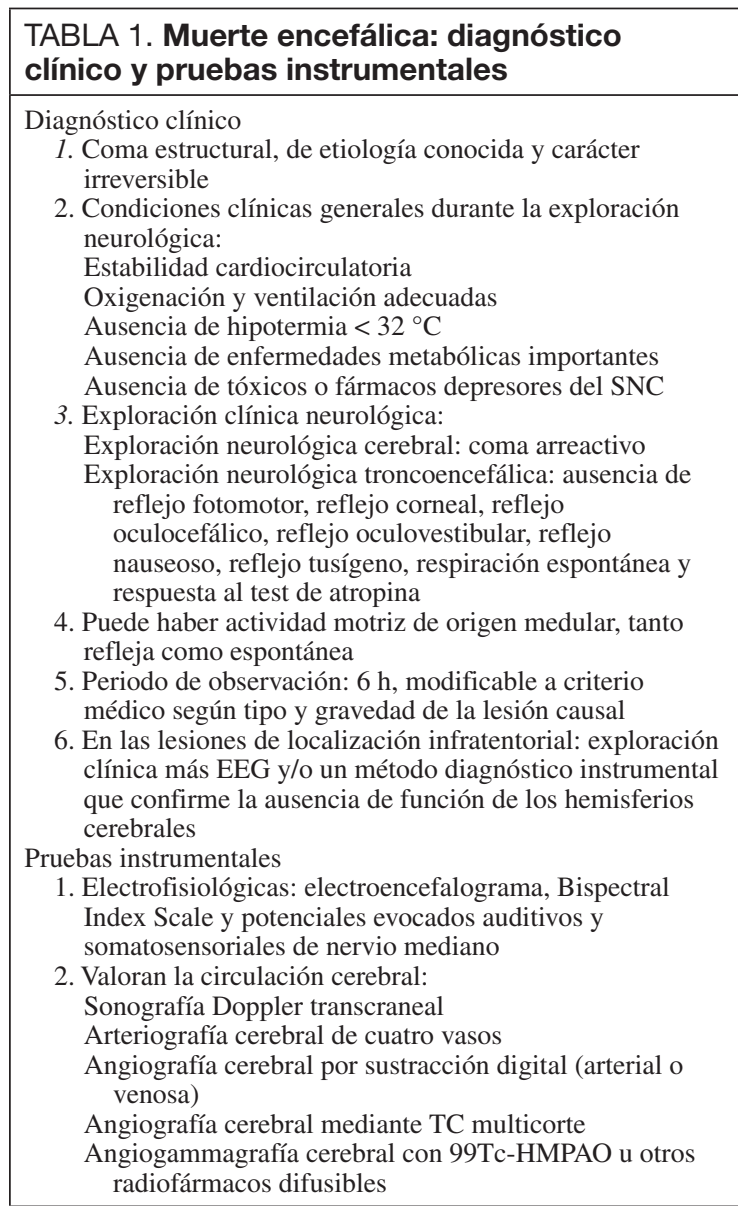

100 corresponde a un individuo despierto, con un nivel de conciencia normal, mientras que una BIS de 0 indica ausencia de actividad eléctrica cerebral. Valores entre 40 y 60 indican un grado adecuado de sedación. Además del valor BIS, el monitor registra también la tasa de supresión (TS), que es el porcentaje de EEG «plano» en el último minuto.

Coincidiendo con el empeoramiento clínico y el aumento de la PIC, se constata un descenso progresivo de la BIS. Los valores BIS caen a 0 inmediatamente después de aparecer la tormenta simpática catecolamínica previa a la parada circulatoria cerebral. Una vez confirmado el diagnóstico de ME, la BIS es 0 y la TS es 100. Puede haber BIS de 0 y reflejo tusígeno, ya que hay un tiempo variable entre la parada circulatoria supratentorial y la pérdida de reflejos troncoencefálicos. Algunas limitaciones de la BIS son que puede dar falsos negativos por hiperpulsatilidad cardíaca y la contaminación electromiográfica ${ }^{22-24}$. Las señales del EEG se recogen en una banda de 0,5-30 $\mathrm{Hz}$, mientras que las señales del electromiograma se encuentran en una banda de 30-300 Hz, lo que justifica que la actividad electromiográfica pueda artefactar los resultados sobrestimando el valor de la BIS. En estos casos, es necesario administrar un bloquea- 

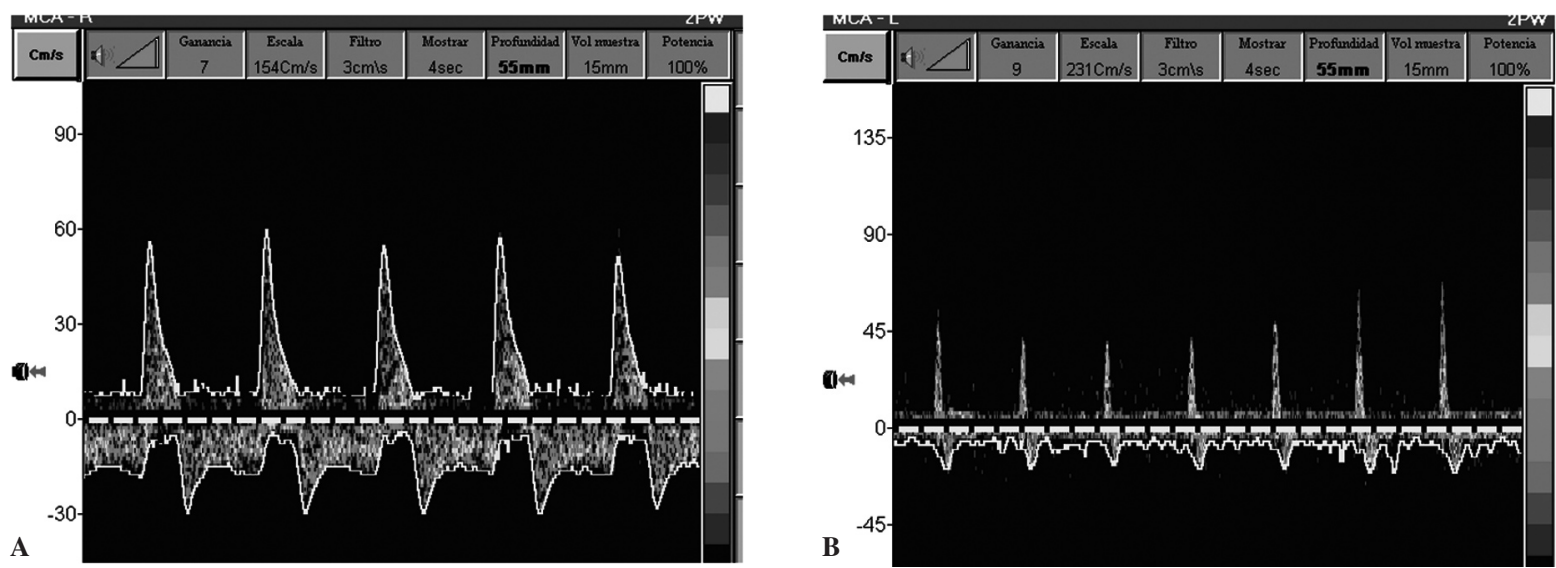

Figura 1. Doppler transcraneal de arteria cerebral media. Patrón de flujo diastólico invertido (A) y de «espigas sistólicas» (B), característicos de parada circulatoria cerebral.

dor neuromuscular para obtener un valor sin interferencias. La ventaja fundamental de la BIS con respecto al EEG convencional es su monitorización continua en tiempo real. Valores de BIS de 0 y TS de 100 deben ser considerados una especie de «semáforo rojo» que alerta sobre la situación del paciente y ayuda a decidir el momento de iniciar el diagnostico de ME. Al ser un estudio regional de la actividad eléctrica, no puede ser utilizado exclusivamente como test confirmatorio de $\mathrm{ME}^{24}$

\section{Potenciales evocados}

Los potenciales evocados (PE) son la respuesta del SNC a un estímulo externo. La estimulación de diversas vías sensitivas provoca una señal eléctrica cortical de muy pequeño voltaje. Según el estímulo sensorial, podemos obtener PE visuales (PEV), PE auditivos troncoencefálicos (PEAT) y PE somestésicos (PES). Los PEAT se obtienen estimulando la vía auditiva, aplicando ruido en forma de clic de $100 \mathrm{~dB}$ y en un número de 1.000-2.000 estímulos en cada oído. El estímulo recorre la vía auditiva desde el VIII par craneal hasta la corteza cerebral auditiva; se registra la actividad eléctrica evocada en los primeros $10 \mathrm{~ms}$ y se generan en este recorrido siete ondas que se corresponden con diferentes estructuras neurológicas. En ME desaparecen todas las respuestas evocadas de origen encefálico, y únicamente persisten las de generación extracraneal. Para el diagnóstico de ME, se utilizan los PES del nervio mediano estudiando los PE generados en los diferentes niveles de la vía sensitiva: nervio periférico, raíz espinal, columna posterior de la médula, lemnisco medio, tálamo contralateral y corteza sensitiva del lóbulo parietal contralateral. En ME sólo persisten las ondas generadas en el plexo braquial y la médula espinal, y desaparecen las ondas de origen intracraneal ${ }^{25,26}$.

Entre las ventajas de los PE, hay que destacar que es una técnica no invasiva que permite monitorizar y seguir la evolución clínica del paciente. Salvo casos muy excepcionales, los $\mathrm{PE}$ no son abolidos por fármacos depresores del $\mathrm{SNC}^{27}$.

\section{Doppler transcraneal (DTC)}

Cuando en un paciente con lesión intracraneal hay un aumento incontrolado de la PIC y paralelamente la presión de perfusión cerebral va disminuyendo, se producen progresivamente un cese del flujo sanguíneo cerebral y la parada circulatoria cerebral. En este proceso pueden distinguirse cuatro etapas ${ }^{28}$.

- La PIC supera la presión arterial diastólica y la velocidad al final de la diástole es cero, pero persiste el flujo durante la sístole. Esta primera etapa no se corresponde todavía con una parada circulatoria completa.

- Sonograma con flujo reverberante, flujo oscilante bifásico o patrón de flujo diastólico invertido (fig. 1A). Se caracteriza por la presencia de flujo anterógrado en sístole, acompañado de un flujo diastólico retrógrado o invertido. Este patrón aparece cuando la PIC es superior o igual a la PAS y se produce el cese de la perfusión cerebral.

- Espigas sistólicas o espículas sistólicas (fig. 1B). En este tipo de patrón se registran únicamente pequeñas ondas sistólicas anterógradas, cortas y puntiagudas, sin obtenerse flujo durante el resto de la sístole ni en la diástole.

- Ausencia de sonograma. No se detecta señal alguna en el DTC. Esta situación aparece en casos muy evolucionados de ME. Para aceptarlo como válido, debe ser confirmado por el mismo explorador que previamente haya observado flujo sanguíneo cerebral. En estos casos, cuando desaparecen las señales intracraneales, se puede realizar el diagnóstico de ME con el estudio de las arterias extracraneales (arterias carótida interna extracraneal, carótida común y vertebrales), ya que también se puede objetivar el patrón de flujo oscilante. Para el diagnóstico de la parada circulatoria cerebral en ME, se recomienda realizar dos exploraciones Doppler separadas al menos unos 
30 min, utilizar el equipo adecuado y que el explorador sea experto en la técnica ${ }^{28-30}$.

Un reciente metaanálisis de trabajos publicados sobre el tema desde 1980 a 2004, sobre un total de 684 pacientes estudiados, refiere una sensibilidad del $89 \%$ y una especificidad del $99 \%$, y llama la atención el elevado número de estudios en que no se realizaba la exploración de la fosa posterior ${ }^{31}$. Para el diagnóstico de ME hay que constatar la parada circulatoria cerebral tanto anterior como posterior.

El DTC es una técnica no invasiva que se puede realizar a pie de cama, permite el control evolutivo del paciente y no tiene interferencia con fármacos depresores del SNC. Su mayor inconveniente es la «mala ventana sónica», que impide el paso de los ultrasonidos hasta en un $10 \%$ de la población, y que da «falsos negativos» en pacientes con drenaje ventricular, craniectomía descompresiva y encefalopatía anóxica ${ }^{30}$. En estos casos, pese a cumplirse los criterios clínicos de ME, persiste flujo en alguna de las arterias intracraneales estudiadas. Aunque no son los hallazgos más habituales, Dosemeci et $\mathrm{al}^{32}$, en un estudio realizado sobre 61 casos con diagnóstico clínico de ME, encuentran persistencia de flujo en $18, \mathrm{y}$ precisan repetir varias exploraciones Doppler hasta 96 h después de la ME clínica para llegar a la confirmación de la parada circulatoria cerebral; refieren una sensibilidad del $70,5 \%$.

\section{Angiogammagrafía cerebral con radiofármacos difusibles}

De los radiofármacos difusibles, uno de los más utilizados es el ${ }^{99} \mathrm{Tc}-\mathrm{HMPAO}$ (hexametilpropilenamina oxima), por su menor coste y su mayor disponibilidad en los servicios de medicina nuclear ${ }^{33-36}$.

Se realiza un control de calidad in vitro para comprobar que el radiofármaco utilizado tiene al menos un $85 \%$ de formas lipofílicas y garantizar la calidad de la técnica. A través de un catéter venoso central, se inyecta el ${ }^{99} \mathrm{Tc}-\mathrm{HMPAO}$ utilizando una dosis de 20 mCi en adultos y al menos $5 \mathrm{mCi}$ en niños.

En proyección anterior se obtiene 1 imagen/s durante el primer minuto tras la inyección. Se estudia el FSC, en el que se observa actividad en ambas arterias carótidas, que van progresando hacia la base del cráneo, el polígono de Willis y finalmente las arterias cerebral media y cerebral anterior. A los 5 min de la fase previa y en proyección anterior, lateral derecha y lateral izquierda, se obtienen las imágenes estáticas o planares. El diagnóstico de $\mathrm{ME}$ se realiza con base en dos criterios: ausencia de perfusión por las arterias carótidas internas durante la fase angiogammagráfica y ausencia completa de captación del radiotrazador en los hemisferios cerebrales y la fosa posterior, imagen que se conoce como el signo del «cráneo hueco» (fig. 2).

La angiogammagrafía cerebral es una técnica con sensibilidad y especificidad del $100 \%$, no tiene interferencia con fármacos depresores del SNC y es poco invasiva, pero es una técnica de uso limitado, ya que no puede realizarse en todos los hospitales.

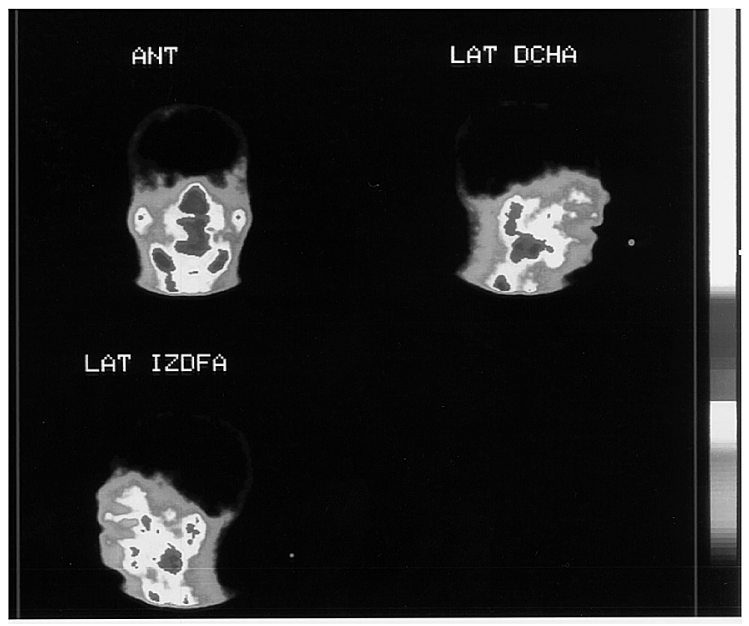

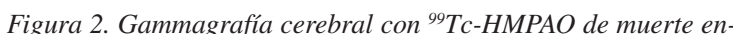
cefálica. Imágenes planares en proyecciones anterior, lateral derecha e izquierda con ausencia de captación parenquimatosa tanto en los hemisferios cerebrales como en la fosa posterior. Se observa el signo del "cráneo hueco» (estudio realizado en el Servicio de Medicina Nuclear II del Hospital Universitario Central de Asturias).

\section{Arteriografía cerebral convencional}

Históricamente se la ha considerado el estándar para demostrar la ausencia de flujo sanguíneo cerebral ${ }^{37}$. Además de la clásica arteriografía de cuatro vasos con cateterización selectiva de las dos arterias carótidas internas (ACI) y las dos arterias vertebrales, se puede realizar una arteriografía cerebral de troncos supraaórticos. En esta técnica, la punta del catéter se sitúa a nivel de la aorta ascendente, donde se inyecta el contraste, con lo que se evitan cambios artificiales en las presiones intraarteriales que pudieran modificar las condiciones reales del flujo sanguíneo cerebral. Igualmente se pueden realizar angiografías por sustracción digital (ASD). La ASD proporciona una mejor sensibilidad de contraste, lo que permite el empleo de concentraciones mucho menores. Las radiografías aumentadas digitalmente son menos detalladas que las imágenes de los angiogramas convencionales, pero las complicaciones son menos frecuentes, ya que no es necesario realizar la cateterización selectiva de las arterias carótidas y vertebrales. Para el diagnóstico de ME, la ASD puede realizarse inyectando el contraste tanto por vía intraarterial como por vía intravenosa ${ }^{38}$. Este tipo de angiografías hoy han sido superadas ampliamente en calidad de imagen y facilidad de la técnica por la angiografía mediante TC helicoidal de alta resolución.

\section{Angiografía cerebral mediante tomografía computarizada (angio-TC)}

En 1998, Dupas et al $^{39}$ describieron en 14 pacientes el diagnóstico de parada circulatoria cerebral mediante angio-TC helicoidal en dos fases. Veinte segundos después de la inyección de contraste, 

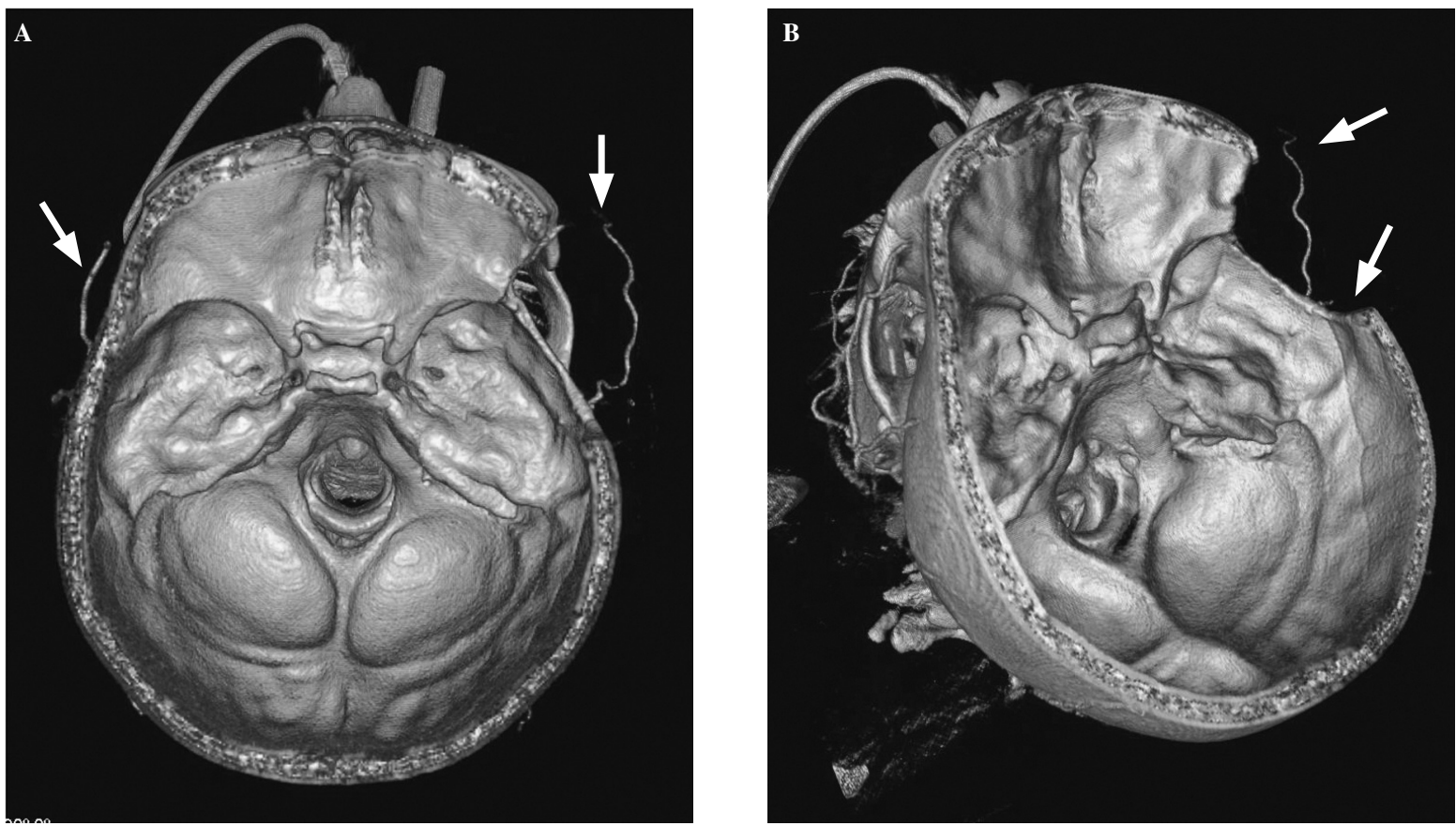

Figura 3. Reconstrucción tridimensional de una angiotomografía computarizada que demuestra la ausencia completa de circulación intracraneal. Se pueden ver las arterias temporales, ramas de la arteria carótida externa y un defecto óseo correspondiente a la craneotomía descompresiva (estudio realizado en el Servicio de Radiología I del Hospital Universitario Central de Asturias).
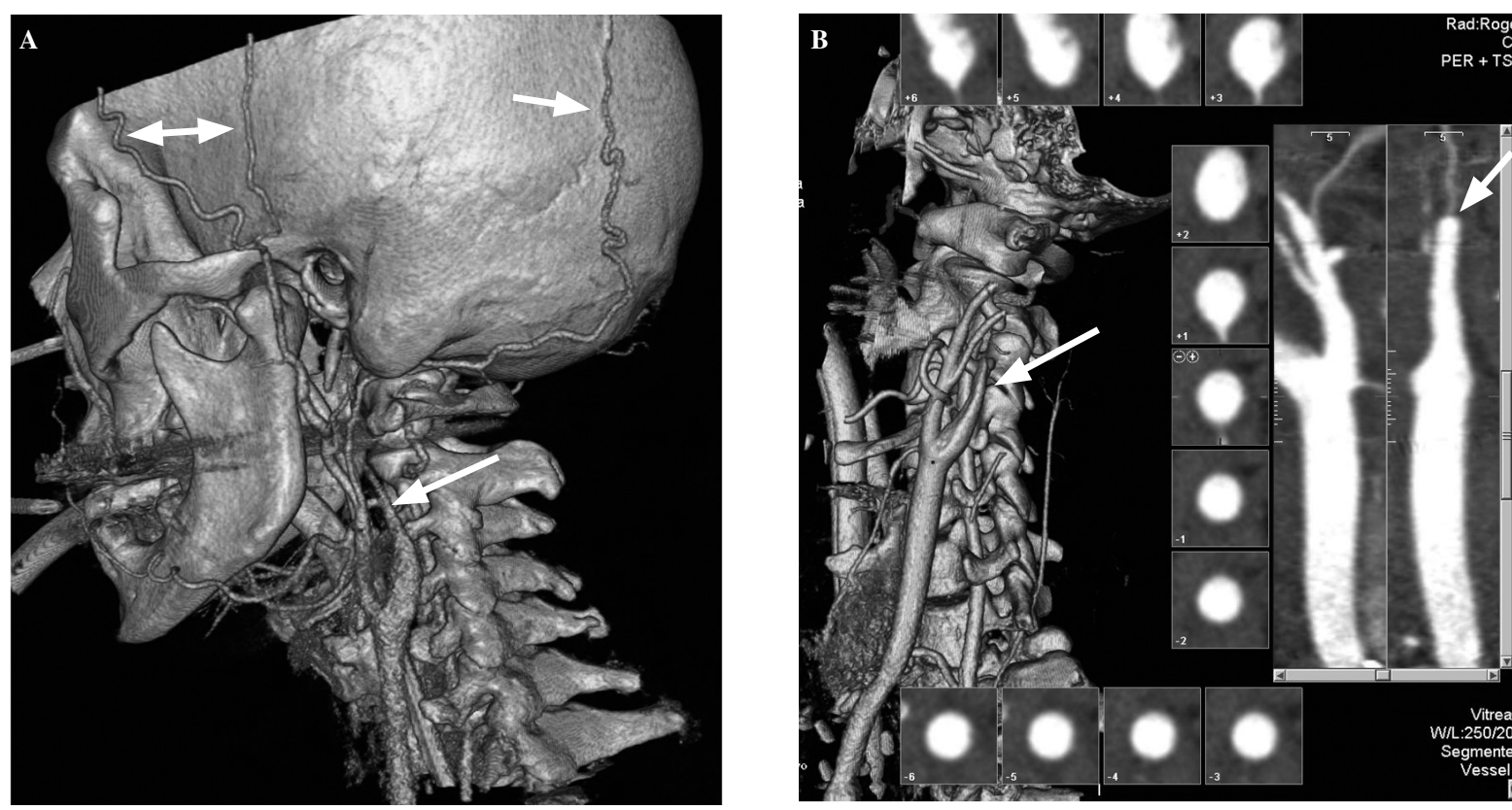

Figura 4. Reconstrucción tridimensional en la que se observa flujo en las arterias carótida común y vertebrales extracraneales, así como en las diversas ramas de la arteria carótida externa. Nótese el afilamiento de la arteria carótida interna (flecha). En el análisis de flujo de los vasos visualizados (marcado mediante línea verde), se observa la desviación del flujo de la carótida primitiva hacia la carótida externa (estudio realizado en el Servicio de Radiología I del Hospital Universitario Central de Asturias).

realizaban una TC para valorar la circulación cerebral arterial, repitiendo la segunda fase a los $54 \mathrm{~s}$ para estudiar la circulación venosa y reconstruían las imágenes cada $5 \mathrm{~mm}$. Los criterios diagnósticos utilizados son la ausencia de contraste en arteria basilar, arterias pericallosas y terminales del córtex, venas profundas, seno sagital superior y seno recto, junto con una visibilidad realzada de la vena oftálmica superior. 
Recientemente, Leclerc et al ${ }^{40}$, publicaron sobre 15 pacientes diagnosticados de parada circulatoria cerebral mediante angio-TC igualmente en dos fases con una TC helicoidal de cuatro detectores. El protocolo técnico y los resultados son similares a los del estudio de Dupas et al, y se concluye que la ausencia de ramas corticales de la ACM y de opacificación de la vena cerebral interna son los mejores criterios para el diagnóstico de ME por angio-TC. Recientemente se han publicado dos trabajos que cuestionan la validez de los criterios diagnósticos establecidos por Dupas et al, ya que se obtiene con esta misma técnica de angio-TC en dos fases un elevado número de falsos negativos por opacificación del segmento A2 de la arteria cerebral anterior y del segmento M4 de la arteria cerebral media. La sensibilidad obtenida oscila en un $52,4-69,7 \%$, y considerándola inaceptable recomiendan un consenso internacional para unificar los criterios radiológicos, y proponen un score basado sólo en la ausencia de opacificación en el retorno venoso a nivel de ambas venas cerebrales internas y gran vena cerebral $1^{41,42}$.

La nueva incorporación de la TC multicorte de alta resolución permite realizar angiografías cerebrales de alta calidad estudiando tanto la circulación anterior a través de arterias carótidas internas como la circulación posterior a través de arterias vertebrales y basilar. Con estas TC multicorte se obtiene una excelente definición de imagen, pueden realizarse reconstrucciones tridimensionales del árbol vascular y se puede diagnosticar la parada circulatoria cerebral. Algunos autores realizan el diagnóstico de ME añadiendo estudios de perfusión cerebral ${ }^{43,44}$.

Nosotros realizamos el estudio en una TC de 64 cortes y reconstruimos las imágenes con un espesor de corte de $0,5 \mathrm{~mm}$. El protocolo radiológico utilizado es el siguiente:

- TC de perfusión: se adquiere un volumen de tejido cerebral a nivel del polígono de Willis y se valora su comportamiento secuencialmente tras la administración de contraste intravenoso. Se realiza el posprocesado habitual en la consola de trabajo analizando la variación en el tiempo de flujo y el volumen sanguíneo cerebral, así como del tiempo medio de tránsito circulatorio y del time-to-peak.

- Angiografía con TC: tras la TC de perfusión, se realiza una angiografía de troncos supraórticos y las arterias cerebrales tras la inyección de $80 \mathrm{ml}(300 \mathrm{mg} /$ $\mathrm{ml}$ a una velocidad de $3,5 \mathrm{ml} / \mathrm{s}$ ) de contraste no ióni$\mathrm{co}$, mediante inyector automático. Se adquiere un volumen de tejido desde el cayado aórtico hasta el vértex. En ME se documenta la ausencia completa de circulación intracraneal tanto anterior como posterior (figs. 3-5). La angiografía cerebral mediante TC multicorte demuestra la ausencia de FSC por los cuatro troncos vasculares y permite diagnosticar la ME de igual forma que hasta ahora se hacía por arteriografía convencional. Esta técnica es más barata, rápida, mínimamente invasiva y de mejor disponibilidad. Su principal indicación es en pacientes sedados o con dificultad para el diagnóstico clínico por uso de barbitúricos u otros fármacos depresores del SNC.

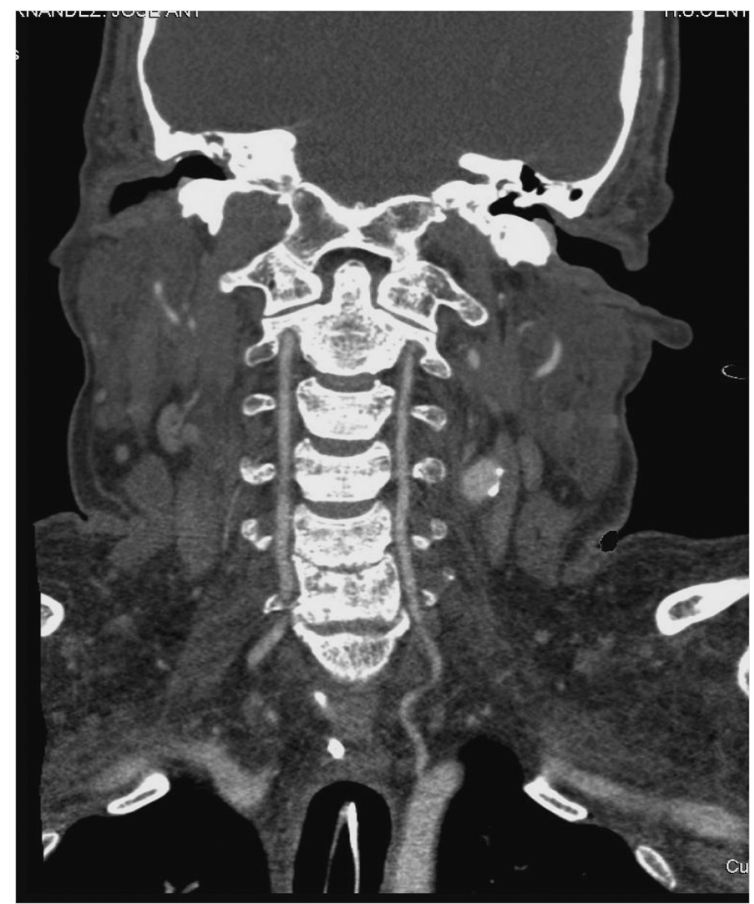

Figura 5. Reconstrucción multiplanar en proyección coronal que deтиеstra relleno de contraste de las arterias vertebrales a nivel extracraneal con ausencia de flujo en la fosa posterior (estudio realizado en el Servicio de Radiología I del Hospital Universitario Central de Asturias).

Pueden verse falsos negativos en pacientes con cráneos abiertos por craniectomía descompresiva y en casos de encefalopatía anóxica. En nuestra experiencia la técnica tiene una sensibilidad del $90 \%$ y ofrece máxima seguridad diagnóstica, con especificidad del $100 \%{ }^{45,46}$.

\section{DECISIONES CLÍNICAS TRAS EL DIAGNÓSTICO DE MUERTE ENCEFÁLICA}

La comunicación de la muerte a la familia debe realizarse de una forma inequívoca y en lenguaje asequible. Hay que informar con claridad que la muerte encefálica es igual al fallecimiento de la persona a efectos científicos, éticos y legales. En España la legislación indica expresamente que la hora del fallecimiento registrada en el certificado de defunción debe ser la hora en que el médico completa el diagnóstico de muerte encefálica ${ }^{1}$. Una vez confirmado el diagnóstico de ME, también es responsabilidad del médico intensivista la toma de decisiones clínicas. En estos casos, y en colaboración con el coordinador de trasplantes, siempre se valorará al paciente como un donante potencial de órganos y se iniciarán las medidas de mantenimiento oportunas. En caso de que la donación de órganos estuviera contraindicada, se procederá a la retirada inmediata de todas las medidas de soporte, incluida la ventilación mecánica. La decisión de retirar las medidas de soporte es responsabilidad del intensivista, y en ningún caso puede estar 
condicionada por la opinión de los familiares ${ }^{47}$. "El individuo no fallece como consecuencia de la retirada de la reanimación, sino justamente a la inversa: se interrumpe la asistencia reanimadora porque el individuo está muerto"48. Como se recoge en la conclusión número 12 de la III Conferencia de Consenso de la SEMICYUC Muerte Encefálica en las Unidades de Cuidados Intensivos, «una vez diagnosticada la ME, se procederá, por parte del médico, a la retirada de las medidas de soporte que se aplicaban al paciente. La obstinación terapéutica, en estos casos, es maleficente y puede ser injusta» ${ }^{11}$. Mantener la ventilación mecánica y un mínimo soporte cardiocirculatorio supone una mala práctica clínica y una inexcusable contradicción con el diagnóstico científico y legal establecido.

\section{BIBLIOGRAFÍA}

1. Real Decreto $2070 / 1999$, de 30 de diciembre, por el que se regulan las actividades de obtención y utilización clínica de órganos humanos y la coordinación territorial en materia de donación y trasplante de órganos y tejidos. BOE 3/2000 de 4-1-2000, p. $179-90$.

2. Escalante JL. Muerte encefálica. Evolución histórica y situación actual. Med Intensiva. 2000;24:97-105.

3. Escalante JL, Escudero D. Introducción. Muerte encefálica en UCI. Med Intensiva. 2000;24:95-6.

4. Escalante JL, Escudero MD, Nolla M, Navarro A y Grupo de Trabajo de Trasplantes de la SEMICYUC. Muerte encefálica en UCI: epidemiología y métodos diagnósticos. En: Libro de Ponencias XXXI Congreso Nacional de la SEMIUC. Castellón, 1996. p. 631-40.

5. Wijdicks EFM. The diagnosis of brain death. N Engl J Med. 2001;344:1215-21

6. Report of the Quality Standards Subcommittee of the American Academy of Neurology. Practice parameters for determining brain death in adults (Summary statement). Neurology. 1995;45:1012-4

7. Escudero Augusto D. Diagnóstico clínico de muerte encefálica. Prerrequisitos y exploración neurológica. Med Intensiva. 2000;24:106-15.

8. Morenski JD, Oro JJ, Tobias JD, Singh A. Determination of death by neurological criteria. J Intensive Care Med. 2003;18: 211-21

9. Wijdicks EFM. Determining brain death in adults. Neurology. 1995;45:1003-11.

10. Escudero D, Otero J. Avances clínicos y legales en el diagnóstico de muerte encefálica durante la década de los trasplantes en España. Nefrología. 2001;XXI:30-40.

11. Conclusiones de la III Conferencia de Consenso de la SEMICYUC. Muerte encefálica en las unidades de cuidados intensivos. Med Intensiva. 2000;24:193-7.

12. Russo H, Bressolle F. Pharmacodynamics and pharmacokinetics of thiopental. Clin Pharmacokinet. 1998;35:95-134.

13. Turcant A, Delhumeau A, Premel-Cabic A, Granry JC, Cottineau C, Six P, et al. Thiopental pharmacokinetics under conditions of long-term infusion. Anesthesiology. 1985;63:50-4.

14. Grattan-Smith PJ, Butt W. Suppression of brainstem reflexes in barbiturate coma. Arch Dis Child. 1993;69:151-2.

15. Pratt OW, Bowles B, Protheroe RT. Brain stem death testing after thiopental use: a survey of UK neurocritical care practice. Anaesthesia. 2006;61:1075-8.

16. Bell D, Moss E, Murphy G. Brainstem death testing in the UK-time for reappraisal? Br J Anaesth. 2004;92:633-40.

17. Cordato DJ, Herkes GK, Mather LE, Gross AS, Finfer S, Morgan MK. Prolonged thiopentone infusion for neurosurgical emergencies: usefulness of therapeutic drug monitoring. Anaesth Intens Care. 2001;29:339-48.
18. Escalante JL, Escudero D. Muerte encefálica. Criterios diagnósticos. En: Ruza F et al, editores. Tratado de cuidados intensivos pediátricos. Madrid: Norma-Capitel; 2002. p. 2069-79.

19. Levesque S, Lessard M, Martin R, Nicole P, Pierre C, Langevin S, et al. Efficacy of a T-piece system and a continuous positive airway pressure system for apnea testing in the diagnosis of brain death. Crit Care Med. 2006;34:2213-6.

20. Frowein RA, Ganshirt H, Richard KE, Hamel E, Haupt WF. Kriterien des Hirntodes: 3.Generation. Anasth Intensivither Notfallmed. 1987;22:17-20.

21. American Clinical Neurophysiology Society. Guideline 3 : Minimum technical standards for EEG recording in suspected cerebral death. J Clin Neurophysiol. 2006;23:97-104

22. Escudero D, Otero J, Muñíz G, Gonzalo JA, Calleja C, Gonzalez A, et al. The Biespectral Index Scale: its use in the detection of brain death. Trasplant Proc. 2005:37:3661-3.

23. Vivien B, Paqueron $X$, Le Cosquer $P$, Langeron $O$, Coriat $\mathrm{P}$, Riou B. Detection of brain death onset using the bispectral index in severely comatose patients. Intens Care Med. 2002;28: 419-25.

24. Escudero D, Otero J, Muñiz G, Parra D, Cofiño L, Taboada F. Detección de muerte encefálica mediante monitorización BIS (Índice Biespectral). Med Intensiva. 2005;29:272-8.

25. Facco E, Machado C. Evoked potentials in the diagnosis of brain death. Adv Exp Med Biol. 2004;550:175-87.

26. Paniagua-Soto J, Piñero Benítez M. Muerte encefálica: ; cuál es el valor diagnóstico de los potenciales evocados multimodales? Med Intensiva. 2000;24:124-34.

27. Schwarz S, Schwab S, Aschoff A, Hacke W. Favorable recovery from bilateral loss of somatosensory evoked potentials. Crit Care Med. 1999;27:182-7.

28. Ducrocq X, Hassler W, Moritake K, Newell D, Von Reutern GM, Shiogai T, et al. Consensus opinion on diagnosis of cerebral circulatory arrest using Doppler-sonografy. Task Force Group on cerebral death of the Neurosonology Research Group of the World Federation of Neurology. J Neurol Sci. 1998;159:145-50.

29. Calleja S, Tembl JI, Segura T, en representación de la SociedadEspañoladeNeurosonología(SONES). Recomendaciones sobre el uso del Doppler transcraneal para determinar la existencia de paro circulatorio cerebral como apoyo diagnóstico de la muerte encefálica. Neurología. 2007;22:441-7.

30. De Freitas GR, Andre C. Sensitivity of transcranial Doppler for confirming brain death: a prospective study of 270 cases. Acta Neurol Scand. 2006;113:426-32.

31. Monteiro LM, Bollen C, Van Huffelen A, Ackerstaff R, Jansen N, Van Vught A. Transcranial Doppler ultrasonography to confirm brain death: a meta-analysis. Intens Care Med. 2006; 32:1937-44

32. Dosemeci L, Dora B, Yilmaz M, Cengiz M, Balkan S, Ramazanoglu A. Utility of transcranial doppler ultrasonography for confirmatory diagnosis of brain death: two sides of the coin. Transplantation. 2004:77:71-5.

33. Munari M, Zucchetta P, Carollo C, Gallo F, De Nardin M, Marzola MC, et al. Confirmatory tests in the diagnosis of brain death: comparison between SPECT and contrast angiography. Crit Care Med. 2005;33:2068-73

34. González FM, De la Riva A, Vallejo JA, Robles JC, Infante de la Torre JR, Delgado F, et al. Medicina nuclear y diagnóstico de muerte encefálica: angiogammagrafía cerebral con Tc99mHMPAO. Sensibilidad y especificidad diagnóstica. Med Intensiva. 2000;24:143-50.

35. Conrad GR, Sinha P. Scintigraphy as a confirmatory test of brain death. Semin Nucl Med. 2003:33:312-23.

36. Al-Shammri S, Al-Feeli M. Confirmation of brain death using brain radionuclide perfusion imaging technique. Med Princ Pract. 2004;13:267-72.

37. Millán JM, Campollo J. Valor diagnóstico de la angiografía cerebral en la confirmación de la muerte encefálica. Ventajas e inconvenientes. Med Intensiva. 2000;24:135-41.

38. Braun M, Ducrocq X, Huot JC, Audibert G, Anxionnat R, Picard L. Intravenous angiography in brain death: report of 140 patients. Neuroradiology. 1997;39:400-5.

39. Dupas B, Gayet-Delacroix M, Villers D, Antonioli D, Veccherine MF, Soulillou JP. Diagnosis of brain death using twophase spiral CT. Am J Neuroradiol. 1998;19:641-7. 
40. Leclerc X, Taschner CA, Vidal A, Strecker G, Savage J, Gauyrit JY, et al. The role of spiral CT for the assessment of the intracranial circulation in suspected brain-death. J Neuroradiol. 2006;33:90-5.

41. Combes JC, Chomel A, Ricolfi F, D’Athis P, Freysz M. Reliability of computed tomographic angiography in the diagnosis of brain-death. Trasplant Proc. 2007;39:16-20.

42. Quesnel C, Fulgencio J-P, Adrie C, Marro B, Payen L, Lembert $\mathrm{N}$, et al. Limitations of computed tomographic angiography in the diagnosis of brain-death. Intens Care Med. 2007; 33:2129-35.

43. Qureshi AI, Kirmani JF, Xavier AR, Siddiqui M. Computed tomographic angiography for diagnosis of brain death. Neurology. 2004;62:652-3.
44. Yu SL, Lo YK, Lin SL, Lai PH, Huang WC. Computed tomographic angiography for determination of brain death. J Comput Assist Tomogr. 2005;29:528-31.

45. Escudero D, Otero J, Vega P, Gil A, Roger R, Gonzalo JA, et al. Diagnóstico de muerte encefálica mediante TC multicorte: angio-TC y perfusión cerebral. Med Intensiva. 2007;31:335-41.

46. Escudero D, Otero J, Marqués L, García E, Iglesias F, Ocampos E, et al. Diagnóstico de muerte encefálica mediante TC multicorte: angio-TC y perfusión cerebral. Med Intensiva. 2007;31:20.

47. Marsé Milla P. Muerte encefálica y toma de decisiones en la unidad de cuidados intensivos. Med Intensiva. 2000;24:189-92.

48. Sociedad Española de Neurología. Diagnóstico neurológico de la muerte cerebral. Dictamen Candanchú 1993. Quaderns CAPS. 1994;20:45-6. 